Chinese Journal of Astronomy and Astrophysics manuscript no.

(LTEX: panagia 'nino $2003 \cdot 2$.tex; printed on July 12, 2018; 3:13)

\title{
The Quest for Primordial Stellar Populations and the James Webb Space Telescope
}

\author{
Nino Panagia * \\ ESA/Space Telescope Science Institute, 3700 San Martin Drive, Baltimore, MD 21218, \\ USA
}

Received 2003 ; accepted 2003

\begin{abstract}
The NASA/ESA/CSA James Webb Space Telescope (JWST) will be the successor to the Hubble Space Telescope and may be launched as early as mid-2011. The key scientific goals for JWST are discovering and understanding the formation of the first stars and galaxies, the evolution of galaxies and the production of elements by stars, and the process of star and planet formation. Within this context, we discuss the expected properties of the first stellar generations in the Universe. We find that it is possible to discern truly primordial populations from the next generation of stars by measuring the metallicity of high-z star forming objects. The very low background of JWST will enable it to image and study firstlight sources at very high redshifts, whereas its relatively small collecting area limits its capability in obtaining spectra of z 10-15 first-light sources to either the bright end of their luminosity function or to strongly lensed sources. With a suitable investment of observing time $J W S T$ will be able to detect individual Population III supernovae, thus identifying the very first stars that formed in the Universe.
\end{abstract}

Key words: space vehicles: instruments — early universe — galaxies: star clusters - supernovae: general

\section{INTRODUCTION}

The James Webb Space Telescope (JWST), formerly the Next Generation Space Telescope $(N G S T)$, is a cooperative program of the National Aeronautics and Space

$\star$ E-mail: panagia@stsci.edu 


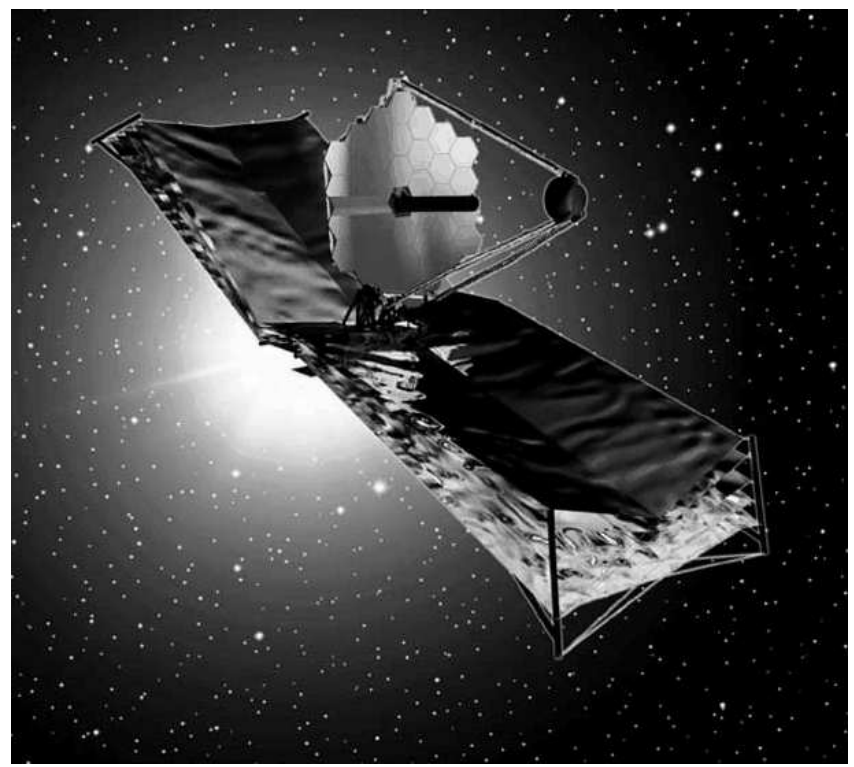

Fig. 1 The Northrop Grumman design for JWST. Easily recognizable are the Optical Telescope Element (OTE), i.e. the cylindrical structure between the primary and the secondary mirror, and the multi-layered deployable sunshield. [Copyright \& Credit: Northrop Grumman Corporation 2000]

Administration $(N A S A)$, the European Space Agency (ESA) and the Canadian Space Agency $(C S A)$ to develop and operate a large, near- and mid-infrared optimized space telescope by the end of this decade that can build and expand on the science opened up by the highly successful Hubble Space Telescope (HST). Jointly, NASA, ESA, and CSA will build JWST, whose construction is to start in 2004 .

JWST has the goal of understanding the formation of galaxies, stars, planets and ultimately, life. JWST is specifically designed for discovering and understanding the formation of the first stars and galaxies, measuring the geometry of the Universe and the distribution of dark matter, investigating the evolution of galaxies and the production of elements by stars, and the process of star and planet formation.

JWST has been under study since 1995 and is planned to be launched around 2011, nearly 400 years after Galileo discovered the moons of Jupiter, over 60 years after Lyman Spitzer proposed space telescopes, and about 20 years after the launch of $H S T$.

$J W S T$ has been conceived as an $6 \mathrm{~m}$ class deployable, radiatively cooled telescope, optimized for the 1-5 $\mu \mathrm{m}$ band, with background limited sensitivity from 0.6 to $10 \mu \mathrm{m}$ or beyond, operating for 10 years near the Earth-Sun second Lagrange point (L2), about 1.5 million km from Earth. It will be a general-purpose observatory, operated by the STScI for competitively selected observers from the international astronomy community.

$J W S T$ is a unique scientific tool, with excellent angular resolution (about 0.05 arcsec at $2 \mu \mathrm{m}$ ) over a large field of view (at least $10 \operatorname{arcmin}^{2}$ ), deep sensitivity and a low infrared 
background. As a cold space telescope, JWST will achieve far better sensitivities than ground-based telescopes. Among the advantages of JWST over potentially competing telescopes are:

- JWST will observe with background levels much lower than possible from the best sites on Earth: for example, the background will be one to six orders of magnitude lower than for Mauna Kea, depending on wavelength, the biggest gain occurring around $5 \mu \mathrm{m}$.

- JWST will have diffraction limited resolution at $2 \mu m$, and will achieve much higher Strehl ratios and wider fields of view than anticipated from ground-based telescopes using adaptive optics.

- JWST's aperture is an order of magnitude larger than SIRTF's, with a factor of 100 better sensitivity.

$J W S T$ will indeed be able to observe the first generations of stars and galaxies, including individual starburst regions, protogalactic fragments, and supernovae out to redshifts of $\mathrm{z}=5-20$. JWST will resolve individual solar mass stars in nearby galaxies, penetrate dust-clouds around star-forming regions, and discover thousands of isolated substellar and Kuiper Belt objects.

\section{THE JWST MISSION CONCEPT}

The science goals for JWST require a telescope with high sensitivity covering the wavelength range from 0.6 to $10 \mu m$, with capability out to $28 \mu \mathrm{m}$, and with NIR resolution comparable to that of HST in the optical. Figure 1 shows the observatory that will be built by the Space Technology division of Northrop Grumman (cleverly named NGST) and will include the Optical Telescope Element (OTE), the Integrated Science Instruments Module (ISIM) Element, and the Spacecraft Element (Spacecraft Bus and Sunshield).

The Integrated Science Instrument Module (ISIM) consists of a cryogenic instrument module integrated with the OTE, and processors, software, and other electronics located in the Spacecraft Support Module (SMM). The instrument suite will include:

- A near IR camera (NIRCam), built by US institutions, covering 0.6-5 $\mu m$, critically sampled at $2 \mu \mathrm{m}$. The field of about $10 \operatorname{arcmin}^{2}$ is apportioned over two sub-cameras each covering a field of $2.3^{\prime} \times 2.3^{\prime}$.

- A near IR multi-object spectrometer (NIRSpec), provided by ESA, with spectral resolutions 1000 and possibly 100, and a spatial resolution of 100 mas, covering a field of view of $3^{\prime} \times 3^{\prime}$ and capable of observing more than 100 objects simultaneously.

- A mid-IR camera/spectrometer (MIRI), built in a 50/50 collaboration between NASA and European institutions, covering a field of 2 ' $\times 2$ ' with a spectral range of $5-28 \mu m$ using a long-slit cross-dispersed grism with a resolution of 1000 .

- A Fine Guidance Sensor (FGS), provided by CSA, that enables stable pointing at the 
milli-arcsecond level and have sensitivity and field of view to allow guiding with $95 \%$ probability at any point on the sky.

The JWST design solve the problem of passively cooling to the cryogenic temperatures required for NIR and MIR operation by (a) protecting the observatory from the Sun with a multi-layer shield, (b) using a heliocentric orbit to decrease the Earth's thermal input, and (c) configuring the telescope to have a large area exposed to space to improve radiative cooling.

With these general characteristics, JWST will have an enormous discovery potential both at 0.6-10 $\mu m$ and at longer wavelengths. In particular, JWST enjoys a considerable background advantage over the ground at all wavelengths, a larger field of view over which high-resolution images can be obtained and a significant aperture advantage over SIRTF. The shorter times required to reach a given threshold can translate into larger fields observed (more targets) and/or greater sensitivities ${ }^{1}$.

\section{PRIMORDIAL STARS: EXPECTED PROPERTIES}

One of the primary science goals of JWST is to answer the question: "When did galaxies begin to form in the early Universe and how did they form?" Theorists predict that the formation of galaxies is a gradual process in which progressively larger, virialized masses, composed mostly of dark matter, harbor star formation as time elapses. These dark-matter halos containing stellar populations, then undergo a process of hierarchical merging and evolution to become the galaxies that make up the local Universe. In order to understand what are the earliest building blocks of galaxies like our own, JWST must detect and identify "first light" sources, i.e, the emission from the first objects in the Universe to undergo star formation.

The standard picture is that at zero metallicity the Jeans mass in star forming clouds is much higher than it is in the local Universe, and, therefore, the formation of massive stars, say, $100 \mathrm{M}_{\odot}$ or higher, is highly favored. The spectral distributions of these massive stars are characterized by effective temperatures on the Main Sequence (MS) around $10^{5}$ K (e.g., Tumlinson \& Shull 2000, Bromm et al. 2001, Marigo et al. 2001). Due to their temperatures these stars are very effective in ionizing hydrogen and helium. It should be noted that zero-metallicity (the so-called population III) stars of all masses have essentially the same MS luminosities as, but are much hotter than their solar metallicity analogues. Note also that only stars hotter than about 90,000 K are capable of ionizing He twice in appreciable quantities, say, more than about $10 \%$ of the total He content (e.g., Oliva \& Panagia 1983, Tumlinson \& Shull 2000). As a consequence even the most massive population III stars can produce HeII lines only for a relatively small fraction of their lifetimes, say, about 1 Myr or about $1 / 3$ of their lifetimes.

\footnotetext{
1 Details and updates can be found at http://www.stsci.edu/jwst/overview//and linked URLs.
} 
The second generation of stars forming out of pre-enriched material will probably have different properties because cooling by metal lines may become a viable mechanism and stars of lower masses may be produced (Bromm et al. 2001). On the other hand, if the metallicity is lower than about $5 \times 10^{-4} \mathrm{Z}_{\odot}$, build up of $\mathrm{H}_{2}$ due to self-shielding may occur, thus continuing the formation of very massive stars (Oh \& Haiman 2002). Thus, it appears that in the zero-metallicity case one may expect a very top-heavy Initial Mass Function (IMF), whereas it is not clear if the second generation of stars is also top-heavy or follows a normal IMF.

\section{PRIMORDIAL HII REGIONS}

The high effective temperatures of zero-metallicity stars imply not only high ionizing photon fluxes for both hydrogen and helium, but also low optical and UV fluxes. This is because the optical/UV domains fall in the Rayleigh-Jeans tail of the spectrum where the flux is proportional to the first power of the effective temperature, $T_{e f f}$, so that, for equal bolometric luminosity, the actual flux scales like $T_{e f f}^{-3}$. Therefore, an average increase of effective temperature of a factor of $\sim 2$ will give a reduction of the optical/UV flux by a factor of $\sim 8$. As a result, one should expect the rest-frame optical/UV spectrum of a primordial HII regions to be largely dominated by its nebular emission (both continuum and lines), so that the best strategy to detect the presence of primordial stars is to search for the emission from associated HII regions.

In Panagia et al. (2003, in preparation) we report on our calculations of the properties of primordial, zero-metallicity HII regions (e.g., Figure 2). We find that the electron temperatures is in excess of $20,000 \mathrm{~K}$ and that $45 \%$ of the total luminosity is converted into the Ly- $\alpha$ line, resulting in a Ly- $\alpha$ equivalent width (EW) of $3000 \AA$ (Bromm, Kudritzki \& Loeb 2001). The helium lines are also strong, with the HeII $\lambda 1640$ intensity comparable to that of $\mathrm{H} \beta$ (Tumlinson et al. 2001, Panagia et al. 2003, in preparation). An interesting feature of these models is that the continuum longwards of Ly- $\alpha$ is dominated by the two-photon nebular continuum. The $\mathrm{H} \alpha / \mathrm{H} \beta$ ratio for these models is 3.2. Both the red continuum and the high $\mathrm{H} \alpha / \mathrm{H} \beta$ ratio could be naively (and incorrectly) interpreted as a consequence of dust extinction even though no dust is present in these systems.

From the observational point of view one will generally be unable to measure a zerometallicity but will usually be able to place an upper limit to it. When would such an upper limit be indicative that one is dealing with a population III object? According to Miralda-Escudé \& Rees (1998) a metallicity $\mathrm{Z} \simeq 10^{-3} Z_{\odot}$ can be used as a dividing line between the pre- and post-re-ionization Universe. A similar value is obtained by considering that the first supernova (SN) going off in a primordial cloud will pollute it to a metallicity of $\sim 0.5 \times 10^{-3} Z_{\odot}$ (Panagia et al. 2003, in preparation). Thus, any object with a metallicity higher than $\sim 10^{-3} Z_{\odot}$ is not a true first generation object. 


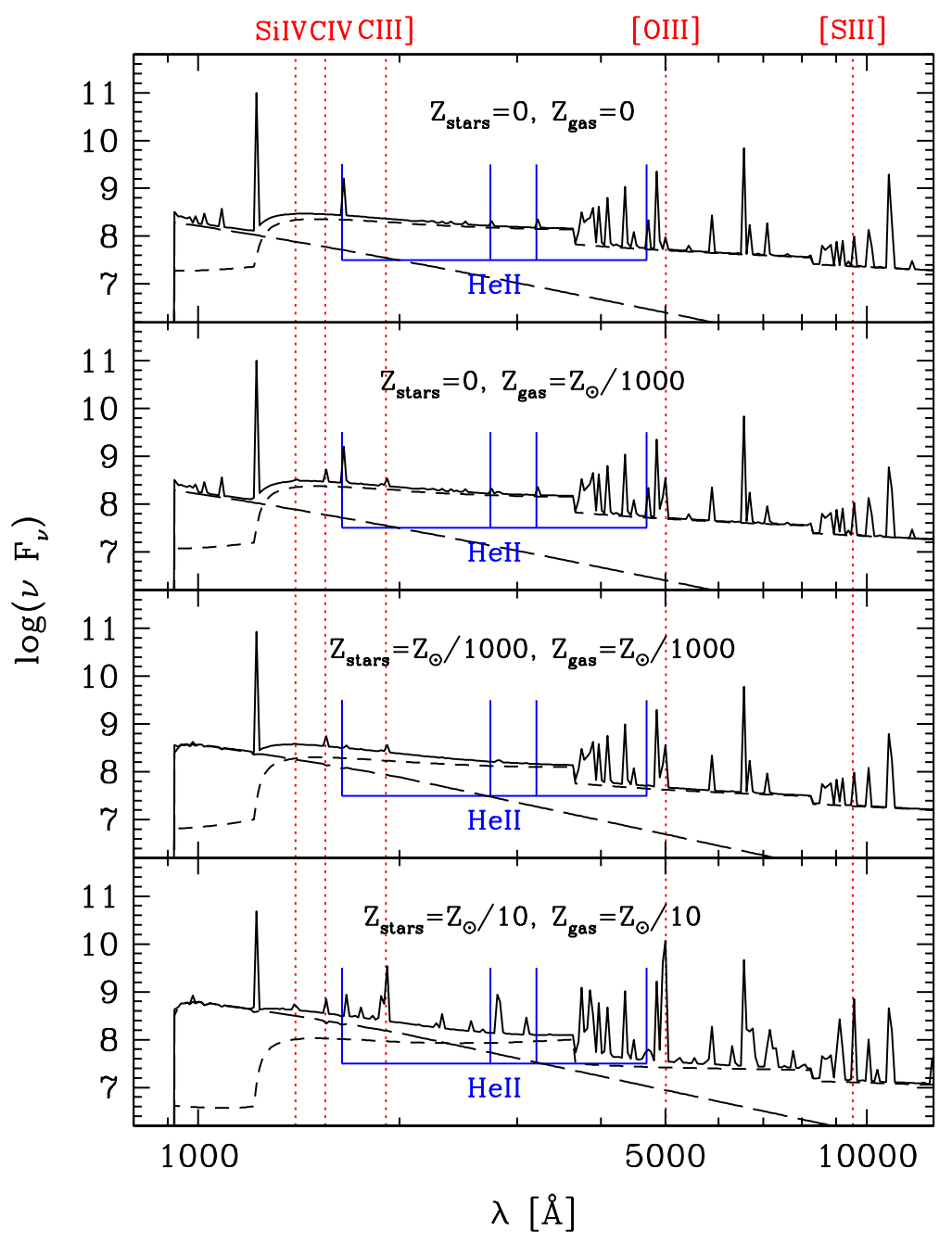

Fig. 2 The synthetic spectrum of a zero-metallicity HII region (top panel) is compared to that of HII regions with various combinations of stellar and nebular metallicities (lower panels). The long-dashed and short-dashed lines represent the stellar and nebular continua, respectively.

\section{LOW METALLICITY HII REGIONS}

We have also computed model HII regions for metallicities from three times solar down to $10^{-6} Z_{\odot}$ (Panagia et al. 2003, in preparation). In Figure 2 the synthetic spectrum of an HII region with metallicity $10^{-3} Z_{\odot}$ (third panel from the top) can be compared to that of an object with zero metallicity (top panel). The two are very similar except for a few weak metal lines. In Figure 3 we show the Ly- $\alpha$ EWs for HII regions ionized by stars with a range of stellar masses and metallicities. Values of EW in excess of 1,000 are possible already for objects with metallicity $\sim 10^{-3} Z_{\odot}$. This is particularly interesting given that Ly- $\alpha$ emitters with large EW have been identified at $\mathrm{z}=5.6$ (Rhoads \& Malhotra 2001). 


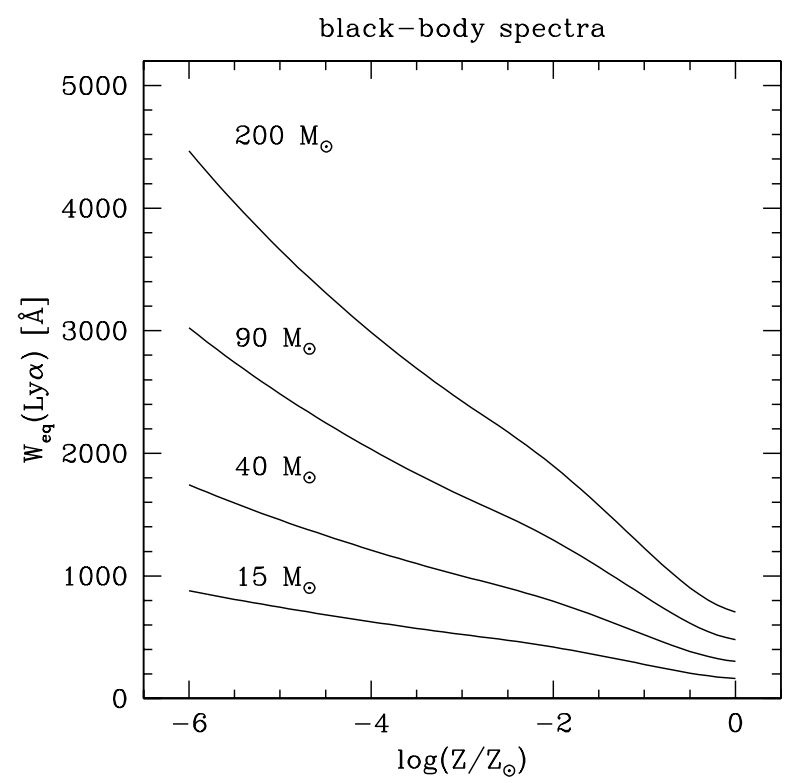

Fig. 3 Ly- $\alpha$ equivalent widths for HII regions ionized by stars with a range of masses and metallicities. The results obtained for black bodies and for stellar atmospheres are very similar to each other.

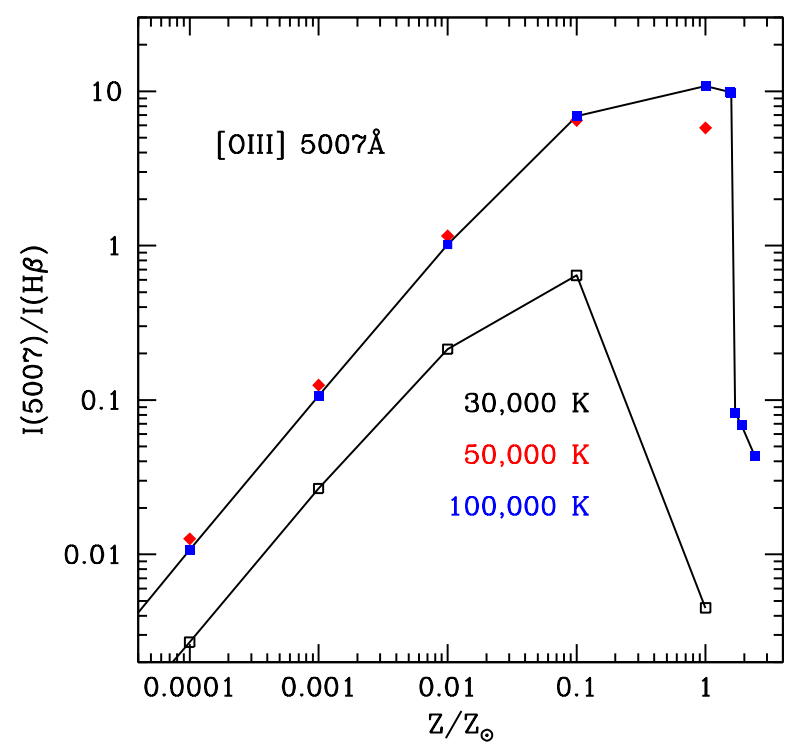

Fig. 4 The ratio [OIII] $] 5007 / \mathrm{H} \beta$ is plotted as a function of metallicity for three different stellar masses: 30,000K (open squares and bottom line), 50,000K (solid diamonds), and 100,000K (solid squares and top line).

The metal lines are weak, but some of them can be used as metallicity tracers. In Figure 4 the intensity ratio of the $[\mathrm{OIII}] \lambda 5007$ line to $\mathrm{H} \beta$ is plotted for a range of stellar temperatures and metallicities. It is apparent that for $Z<10^{-2} Z_{\odot}$ this line ratio traces metallicity linearly. Our reference value $Z=10^{-3}$ corresponds to a ratio [OIII] $/ \mathrm{H} \beta=0.1$. 


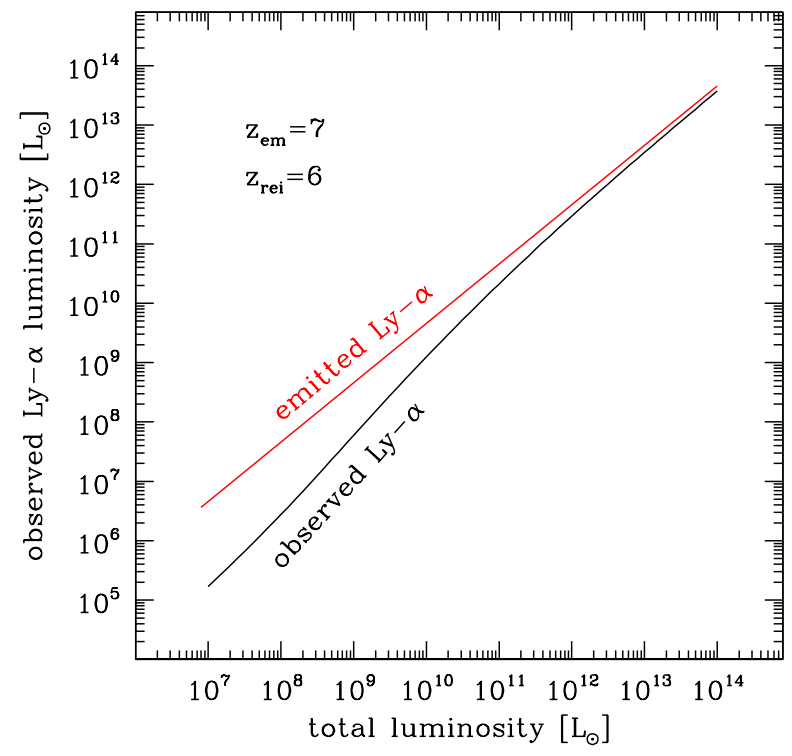

Fig. 5 Transmitted Ly- $\alpha$ intensity as a function of the object luminosity. Bright objects ionize their neighborhood and are able to reduce the Ly- $\alpha$ attenuation. labelfig 5

The weak dependence on stellar temperature makes sure that this ratio remains a good indicator of metallicity also when one considers the integrated signal from a population with a range of stellar masses.

Another difference between zero-metallicity and low-metallicity HII regions lies in the possibility that the latter may contain dust. For a $Z=10^{-3} Z_{\odot}$ HII region dust may absorb up to $30 \%$ of the Ly- $\alpha$ line, resulting in roughly $15 \%$ of the energy being emitted in the far IR (Panagia et al. 2003, in preparation).

\section{HOW TO DISCOVER AND CHARACTERIZE PRIMORDIAL HII REGIONS}

It is natural to wonder whether primordial HII regions will be observable with the generation of telescopes currently on the drawing boards. In this section we will focus mostly on the capabilities of the James Webb Space Telescope.

Before proceeding further we have to include the effect of HI absorption in the IGM on the Ly- $\alpha$ radiation (Miralda-Escudé \& Rees 1998, Madau \& Rees 2001, Panagia et al. 2003, in preparation). A comparison of the observed vs emitted Ly- $\alpha$ intensities is given in Figure 5. The transmitted Ly- $\alpha$ flux depends on the total luminosity of the source since this determines the radius of the resulting Strömgren sphere. A Ly- $\alpha$ luminosity of $\sim 10^{10} \mathrm{~L}_{\odot}$ corresponds to $\sim 10^{6} \mathrm{M}_{\odot}$ in massive stars. In the following we will consider this as our reference model. 


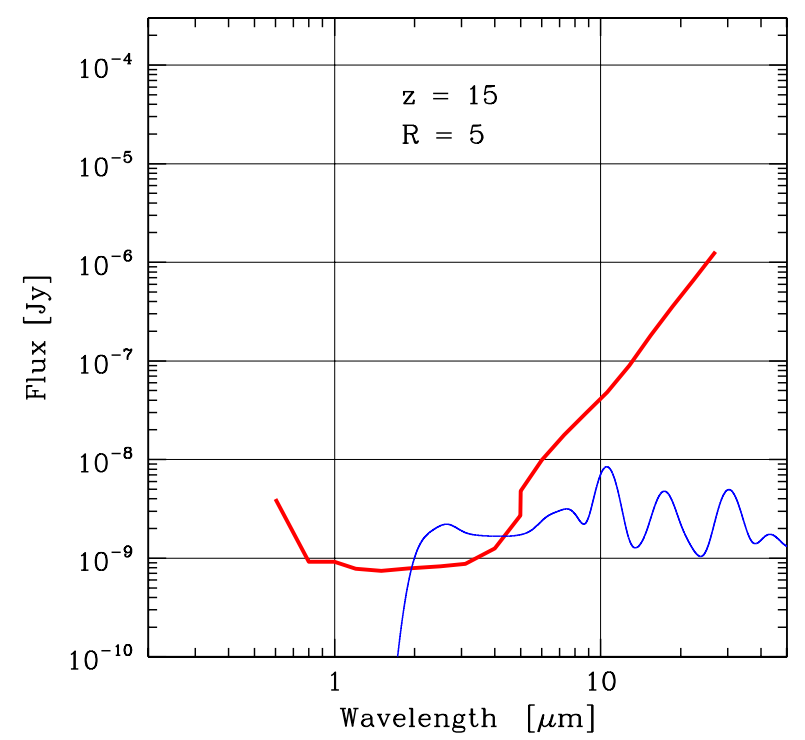

Fig. 6 Synthetic spectral energy distribution of a $Z=10^{-3} Z_{\odot}$ starburst object at $\mathrm{z}=15$ containing $10^{6} \mathrm{M}_{\odot}$ in massive stars (thin line) compared to the imaging limit of $J W S T$ at $\mathrm{R}=5$ (thick line). The $J W S T$ sensitivity refers to $4 \times 10^{5} \mathrm{~s}$ exposures with $\mathrm{S} / \mathrm{N}=10$.

The synthetic spectra, convolved with suitable filter responses can be compared to the $J W S T$ imaging sensitivity for $4 \times 10^{5} \mathrm{~s}$ exposures (see Figure 6). It is clear that JWST will be able to easily detect such objects. Due to the high background from the ground, JWST will remain superior even to $30 \mathrm{~m}$ ground based telescopes for these applications.

The synthetic spectra can also be compared to the JWST spectroscopic sensitivity for $4 \times 10^{5} s$ exposures (see Figure 7). It appears that while the Ly- $\alpha$ line can be detected up to $z \simeq 15-20$, for our reference source only at relatively low redshifts $(\mathrm{z} \sim 7)$ can $J W S T$ detect other diagnostics lines lines such as HeII 1640A, and Balmer lines. Determining metallicities is then limited to either lower redshifts or to brighter sources.

We can reverse the argument and ask ourselves what kind of sources can JWST detect and characterize with spectroscopic observations. Figure 8 displays, as a function of redshift, the total luminosity of a starburst whose lines can be detected with a $\mathrm{S} / \mathrm{N}=10$ adopting an exposure time of $4 \times 10^{5} \mathrm{~s}$. The loci for Ly- $\alpha, \mathrm{HeII} 1640 \AA, \mathrm{H} \beta$, and [OIII] $5007 \AA$ are shown. It appears that Ly- $\alpha$ is readily detectable up to $\mathrm{z} \simeq 20$, HeII $1640 \AA$ may also be detected up to high redshifts if massive stars are indeed as hot as predicted, whereas "metallicity" information, i.e. the intensity ratio $\mathrm{I}([\mathrm{OIII}]) / \mathrm{I}(\mathrm{H} \beta)$, can be obtained at high redshifts only for sources that are 10-100 times more massive or that are 10-100 times magnified by gravitational lensing. 


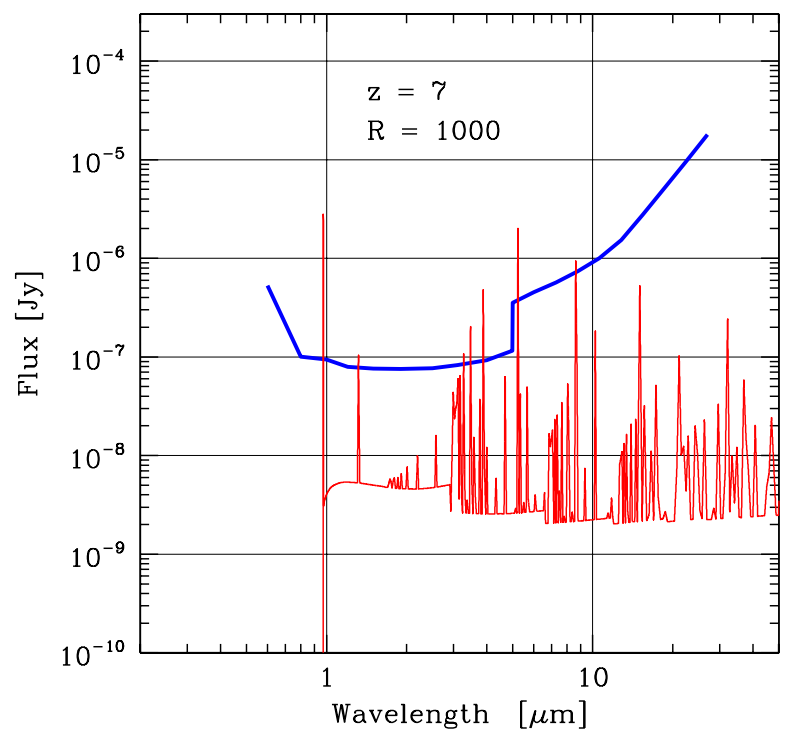

Fig. 7 Synthetic spectrum of a $\mathrm{Z}=10^{-3} Z_{\odot}$ starburst object at $\mathrm{z}=7$ containing $10^{6} \mathrm{M}_{\odot}$ in massive stars (thin line) compared to the spectroscopic limit of JWST at $\mathrm{R}=1000$ (thick line). The $J W S T$ sensitivity refers to $4 \times 10^{5} \mathrm{~s}$ exposures with $\mathrm{S} / \mathrm{N}=10$.

\section{PRIMORDIAL SUPERNOVAE}

Even if JWST cannot detect individual massive Population III stars, supernova explosions may come to the rescue. We know that local Universe supernovae (SNe) can be as bright as an entire galaxy (e.g., at maximum light Type Ia supernovae, or SNIa, have $\left.\mathrm{M}_{B}(S N I a) \simeq-19.5\right)$ and as such, they may be detectable up to large distances. Practically, SNIa are efficient emitters only at rest frame wavelengths longer than 3000A, and, therefore, they will be hard to detect at redshifts higher than $\mathrm{z} \simeq 10$ (Panagia 2003a,b). Moreover, the stellar evolution that leads to classical SNIa explosions is believed to take several hundred million years (e.g., Madau, Della Valle \& Panagia 1998) and, therefore, no type Ia is likely to occur at redshifts higher than about $\mathrm{z} \simeq 8$. Type II suypernovae (SNII) are much more efficient UV emitters but only rarely they are as bright as a SNIa. As a consequence, they will barely be detected at redshifts higher than 10 (Panagia 2003a,b) , or, if they are exceptionally bright (the so-called supernovae of type IIn, à la SN 1979C or SN 1998S) they would be rare events.

On the other hand, massive population III stars are much more massive than Pop II or Pop I stars, and the resulting supernovae may have properties very different from those of local Universe SNe. Heger et al (2001) have considered the fate of massive stars in conditions of zero metallicity and have found that stars more massive than $260 \mathrm{M}_{\odot}$ would directly collapse to a black hole without producing an explosion, as well as stars with masses in the approximate range $30-140 \mathrm{M}_{\odot}$. Below $30 \mathrm{M}_{\odot}$ the SN explosions would 


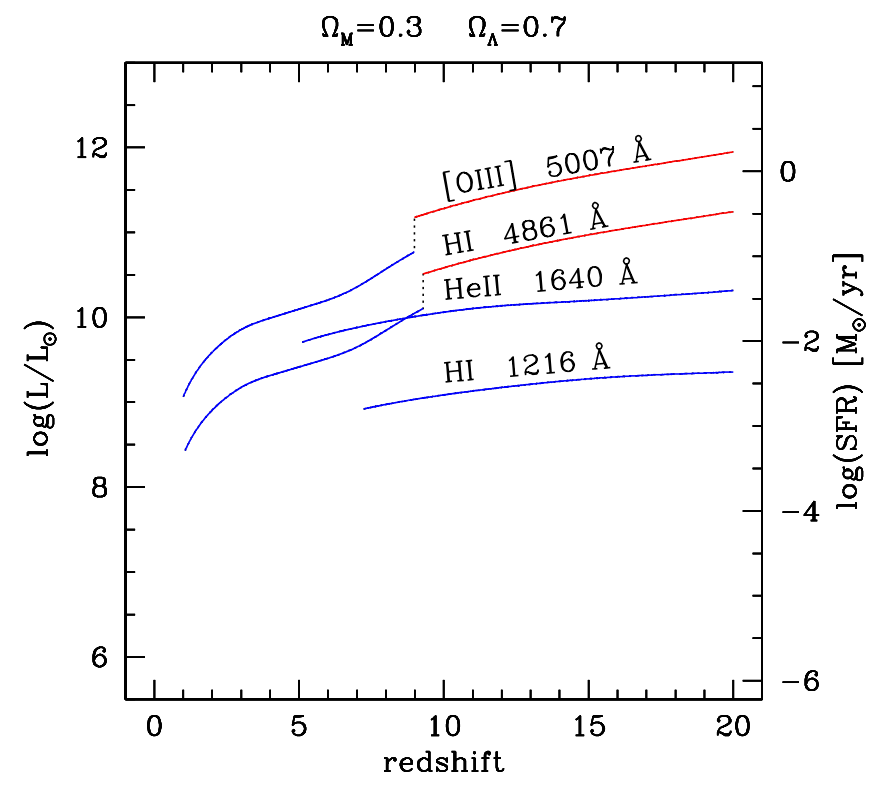

Fig. 8 Limiting total luminosity of the ionizing stars (left-hand scale) and topheavy IMF star formation rate (right-hand scale) to detect various emission lines using JWST spectroscopy, with $\mathrm{S} / \mathrm{N}=10$ in integrations of 100 hours, as a function of the source redshift.

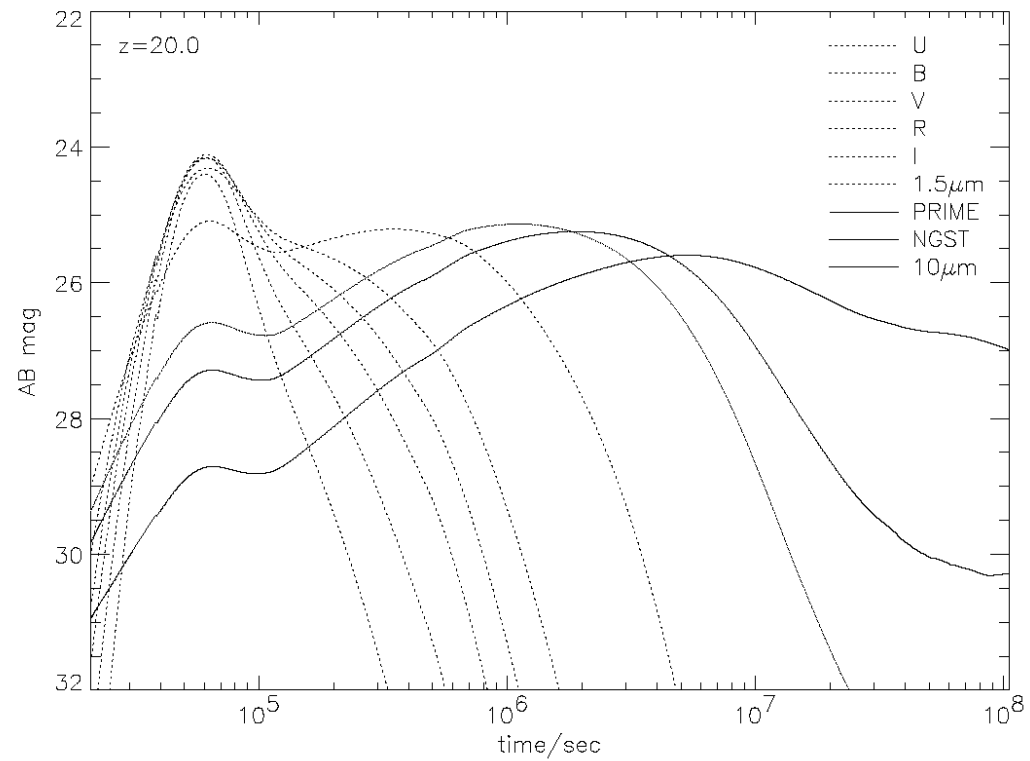

Fig. 9 Light curves for a pair-creation supernova from a $250 \mathrm{M}_{\odot}$ star at $\mathrm{z}=20$ (Heger et al. 2001). Time, wavelengths and magnitudes are given in observer rest-frame. Wavelengths shorter than the IGM Ly-a absorption $(2.55 \mu \mathrm{m})$ are shown as dotted lines. The curves labelled as "PRIME" and "NGST" correspond to observed wavelengths of 3.5 and $5 \mu \mathrm{m}$, respectively. 
resemble those of type II supernovae $\left(\mathrm{E}_{k i n} \simeq 1 \times 10^{51} \mathrm{erg}\right)$. For stellar progenitors with masses in the range 140-260 $\mathrm{M}_{\odot}$, Heger et al. find that the explosions would be caused by a pair-production instability and would be 3 to 100 times more powerful than than core-collapse (Type II and Type Ib/c) SNe. As a consequence, even an individual SN may become bright enough to be detected with JWST. In particular, Heger et al calculate that near maximum light the brightest pair-production $\mathrm{SNe}$ at a redshift of $\mathrm{z}=20$ may be observed at a flux level of about $100 \mathrm{nJy}$ at $5 \mu \mathrm{m}$, or, correspondingly, be brighter than an AB magnitude of 26. This high flux is more than 100 times brighter than that of a typical SNII and, therefore, would be well within the reach of JWST observations made with an integration time of a few hours.

While bright supernovae produced by the explosions of primordial Pop III stars may be "detectable", do they occur frequently enough to be found in a systematic search? For a standard cosmology $\left(\Omega_{\Lambda}=0.7, \Omega_{m}=0.3, \mathrm{H}_{0}=65 \mathrm{~km} \mathrm{~s}^{-1} M p c^{-1}, \Omega_{b}=0.047\right)$, and assuming that at $\mathrm{z}=20$ a fraction $10^{-6}$ of all baryons goes into stars of $250 \mathrm{M}_{\odot}$, Heger et al. (2001) predict an overall rate of 0.16 events per second over the entire sky, or about $3.9 \times 10^{-6}$ events per second per square degree. Since for these primordial SNe the first peak of the light curve lasts for about a month (see Figure 9), about a dozen of these supernovae per square degree should be at the peak of their light curves at any time. Therefore, by monitoring about 100 NIRCam fields with integration times of about 10,000 seconds at regular intervals (every few months) for a year should lead to the discovery of three of these primordial supernovae. We conclude that, with a significant investment of observing time (a total of 4,000,000 seconds) and with a little help from mother Nature (to endorse our theorists views), JWST will be able to detect individually the very first stars and light sources in the Universe.

\section{References}

Baraffe I., Heger A., Woosley S. E., 2001, ApJ, 550, 890

Bromm V., Ferrara A., Coppi P. S., Larson R. B., 2001, MNRAS, 328, 969

Bromm V., Kudritzki R. P., Loeb, A., 2001, ApJ, 552, 464

Gunn J. E., Peterson B. A., 1965, ApJ, 142, 1633

Heger A., Woosley S. E., 2002, ApJ, 567, 532

Heger A., Woosley S. E., Baraffe I., Abel T., 2001, astro-ph 0112059

Izotov Y. I., Thuan T. X., 1998, ApJ, 500, 188

Madau P., Della Valle M., Panagia N., 1998, MNRAS 297, L17

Madau P., Rees M. J., 2001, ApJ, 551, L27

Marigo P., Girardi L., Chiosi C., Wood P. R. 2001, A\&A 371, 252

Miralda-Escudé J., Rees M. J., 1998, ApJ, 497, 21

Oh S. P., Haiman Z., 2002, ApJ, 569, 558

Oliva E., Panagia N., 1983, Ap\&SS, 94, 437

Panagia N., 2003a, in "Supernovae and Gamma-Ray Bursts", ed. K. W. Weiler (SpringerVerlag), p. 113-144

Panagia N., 2003b, STScI Newsletter, Vol. 20, Issue 4, in press

Rhoads, J. E., Malhotra S., 2001, ApJ, 563, L5 
Tumlinson J., Shull J. M., 2000, ApJ, 528, L65

Tumlinson J., Giroux M. L., Shull J. M., 2001, ApJ, 550, L1 